\title{
Accuracy Measurements of Miniature Robot Using Optical CMM
}

\author{
Asser Vuola and Reijo Tuokko \\ Tampere University of Technology, Department of Production Engineering \\ Korkeakoulunkatu 6, 33720 Tampere, Finland \\ \{asser.vuola, reijo.tuokko\} atut.fi \\ http://www.tut.fi/tte
}

\begin{abstract}
This paper describes accuracy measurement method for high resolution miniature robot manipulators. The robot itself, measuring method and the results are presented. Parallel kinematic robot structures have by now seen as the most promising, due to their simpler and stiffer structure and easier miniaturisation. A robot using the direct drive method and direct high resolution feedback has been studied. The accuracy measurement for such robot is not straightforward because of the sub micrometre accuracy requirements and robots incapability to carry any measurement object or sensor. New kind of method is presented in this paper. It bases on the use of non-touching optical Coordinate Measuring Machine (CMM). Main advantage of presented method is its capability to traceable measurements and minimal interference with the measured robot. Results show that presented method is suitable for the accuracy measurements of the miniature devices.
\end{abstract}

Keywords: desktop factory, micro factory, parallel robot, accuracy measurement, repeatability.

\section{Introduction}

This paper describes accuracy measurement method for high resolution miniature robot manipulators. The robot itself, measuring method and the results are presented. Motivation for this research is the lack of suitable measurement method. Typical methods are usually stationary sensor setups (optical, capacitive etc.). Some type of measurement target is usually required to be mounted on the robot. Focus in the design of the presented robot has been the demonstration of the concept of the small parallel scara type robot using direct drive motors and direct feedback. Due to the relatively low forces generated by the motors leads to the situation that heavy measurement target cannot be used. Typical methods are also capable only measuring repeatability, not accuracy.

The use of coordinate measuring machine is promising idea, due to their extremely high accuracy and traceability of the measurements. Coordinate Measuring Machines (CMM) are usually based on touching probe principle. This method is not suitable, because it is strongly affecting the robot to be measured. CMM cannot be used with 
traditional size robots as the robot cannot be installed in the machine. Situation with the micro robot is quite opposite, as complete robot cell can be easily placed inside the CMM.

\section{Parallel Scara Robot}

Manipulator is the key issue in the assembly system and the most demanding part in the miniaturisation process. Conventional assembly manipulator is typically cartesian, scara- or arm-type robot. Common problem of all these type of mechanisms is the scalability of the moving mass relatively to the performance values. Many practical difficulties like cabling, mountings, friction, etc. also occur when one is trying to minimise such structures. Most critical performance deterioration is the decrease of the relative repeatability, caused by the hysteresis in the pinion drives and bearings. The use of parallel kinematics in smaller scale robots has been seen very promising method to overcome these issues. Parallel robots are closed loop mechanisms presenting very good performances in terms of accuracy, velocity and rigidity compared to their own size and mass. Fig. 1 show miniature test robots using parallel kinematics and figure show the workspace of the robot. [1]

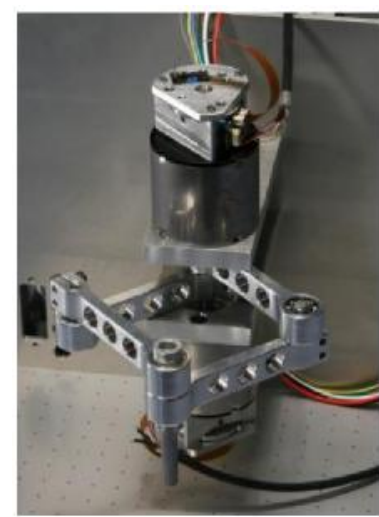

Fig. 1. Paraller test robot

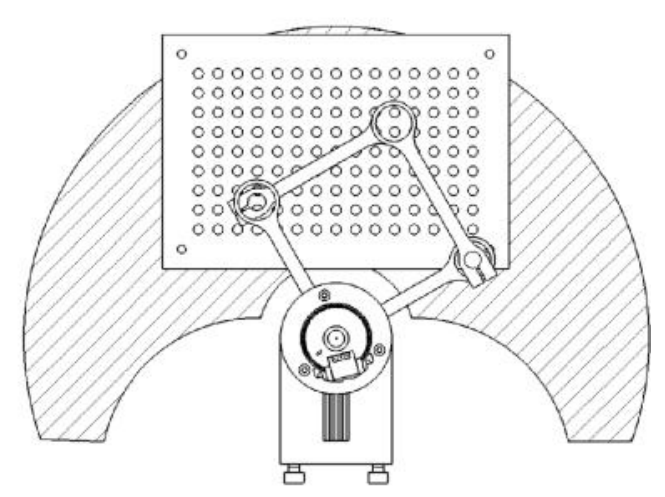

Fig. 2. Workspace of the test robot

The mass of the actuators comes more dominant compared to the mass of the whole device when system size goes smaller, especially if sufficient high accuracy is demanded. Therefore the main advantage of parallel kinematics in small scale devices is that the actuators are not a part of the moving mass. Suitable gear for the high accuracy micro robots is harmonic drive. Use of harmonic drive along with the miniature motors in the micro robot is successfully demonstrated with the Parvus robot [3]. As there were some major problems in demonstrating the H-Scare robot, only a simple test robot is built using direct drive technology. Direct drive has been successfully used in many delta kinematic robots. Thus it is selected for this robot to test its applicability in parallel scara robot. Mechanical structure contains four 
identical joints which are coincidence in both ends of the structure. This guarantees the largest workspace. As predicted, the robot is extremely fast, which is obvious consequence from the direct drive structure. However, servo system PID-tuning appeared to be challenging. This leads to the situation that the robot is either very soft/flexible or unstable and noisy. As high accuracy is the key factor, relatively soft tuning is selected to measure maximum accuracy for the structure with selected drive and feedback.

$15 \times 9$ size grid with $7 \mathrm{~mm}$ spacing is fitted to the working area of the robot. Grid is pick and place demo for small steel balls but the same grid is used in accuracy measurements as positioning could easily be visually checked during the measurements. Figure 1 and 2 presents the robot, test grid and the working area of the robot. Z-axis is implemented with a ball screw driven axis. As it is very traditional technology and not interfering with the parallel structure, which is the main interest of this paper, only planar motions with parallel arms are studied in the accuracy measurements.

\section{$3 \quad$ Measurement Method}

The measurement method bases on measuring position of the end point of robot arms with optical CMM over the robot's work area. Robot is driven to different positions in matrix while CMM measures the actual position of the tip of robot. Each point is repeated several times and approach directions. Accuracy of measurement is increased by use of special target. Target itself requires special procedure at CMM to find firstly the rough position mark and secondly three ruby balls to increase accuracy. The details of the method are opened in the following chapters.

\subsection{Optical CMM}

Measurement method is built around the Mahr OMS 1000 coordinate measurement machine. A technical specification of the machine is:

- Granite portal type body (8 tons) with moving table (Y-axis)

- Measuring Volume: $\left(1000\right.$ x 1000 x 600) $\mathrm{mm}^{3}$

- Volumetric Accuracy: $\mathrm{U} 3= \pm(2.2+\mathrm{L} / 300) \mu \mathrm{m}$, as $\mathrm{L}=$ measuring distance in $\mathrm{mm}(\mathrm{k}=2)$ according to VDI/VDE 2617 length measuring test

- Sensors: Two CCD-cameras: reference camera and another with 0.5x smaller magnification, and two lenses $3 \mathrm{x}$ and 10x. Corresponding field of view (FOV) are $4 \times 3 \mathrm{~mm}$ and $1,2 \times 0,9 \mathrm{~mm}$.

All axes have air bearings and ball screw drives. Direct position measurement is made using glass bar linear encoder. Calibration of the machine is maintained regularly, according to the traceability requirements. Figures 3 and 4 present the CMM machine and the measuring setup. 


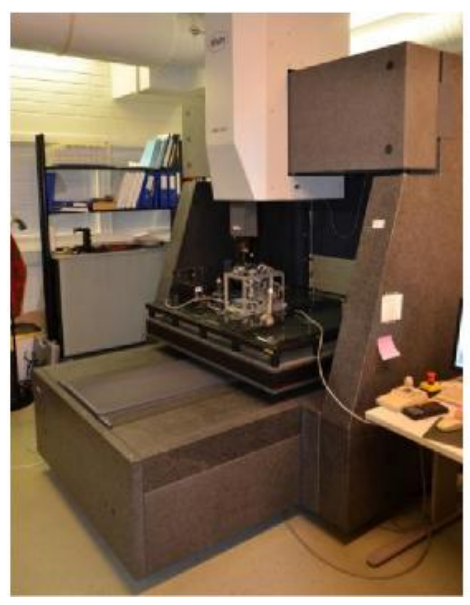

Fig. 3. Mahr OMS 1000 CMM

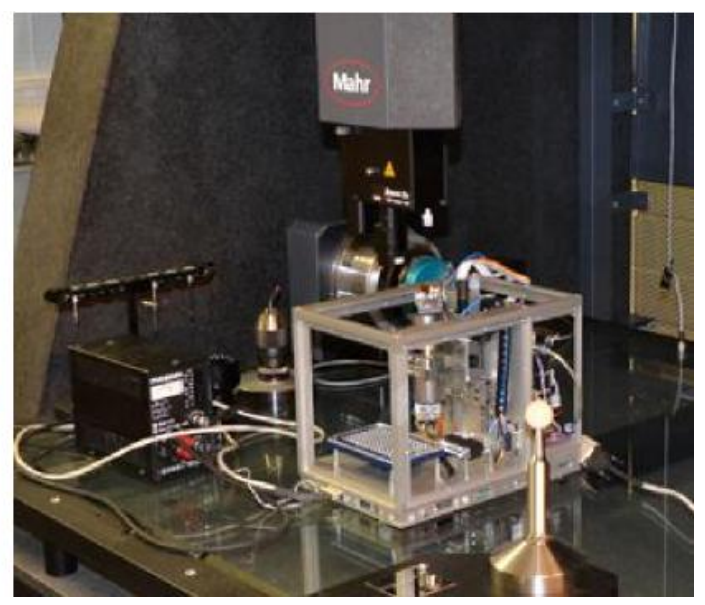

Fig. 4. Measurement setup

Machine has two measuring methods: optical (camera based) and touching probe. Optical measuring system is used as touching probe method would significantly interfere with the robot. There is a major doubt towards the reliability of the optical method as it cannot be considered exact. Optical coordinate measuring bases on several line- and circle- fitting machine vision algorithms and thus the result is depending on the lighting conditions as well as other environmental aspects [3]. Uncertainty of the measurement method could be approximated by combining different error sources. Uncertainty budget is presented in table 1. All uncertainties are presented as range, because uncertainty is function of distance of the CMM from its origin, excluding the most unstable corner positions.

Table 1. Uncertainty budget of the measurement

\begin{tabular}{clc}
\hline & Uncertainty component & Standard uncertainty $(\mu \mathrm{m})$ \\
\hline$\delta \mathrm{d}_{\mathrm{CMM}}$ & CMM positioning error & $2,2-2,6$ \\
$\delta \mathrm{d}_{\mathrm{r}}$ & Repeatability error of one ruby & $0,5-1,5$ \\
$\delta \mathrm{d}$ & Rep. error of one measurement & $3,0-5,0$ \\
\hline
\end{tabular}

\subsection{Measurement Target}

Easiest way for robot accuracy measurements would be the direct measuring from the structure of the end effector of the robot. Parallel joints of the robot are attached with $6 \mathrm{~mm}$ diameter axle. The first idea was to measure the centre point of the axle, but as it is challenging to manufacture metal parts with sharp edges, the edge finding of the circular axis is not an accurate method. This was clearly pointed out by preliminary tests. Repeatability of several measurements was only $20 \mu \mathrm{m}$, which is far too much for this purpose. Laser marked target object at end surface of the axle was tested next, but the result were even worse. Third option was to use ruby ball as target for optical 
measurement. A $2 \mathrm{~mm}$ diameter probe ball from Renishaw Company was selected. As the orientation of the robot is also interesting, several balls must be used. Three ruby balls are arranged to triangular positions. Laser marked figure at the end of the robot axle is used for pre-alignment. This shape helps to automate measuring process. Figure 5 and 6 presents the target and its dimensions. Green light in figure 5 is the coaxial illumination of the camera system. Pre-alignment shape on the top of the axis can be seen on camera image in figure 7 .

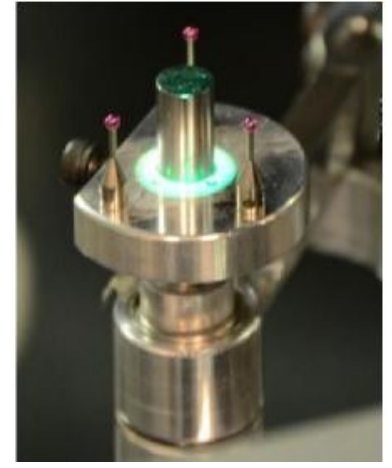

Fig. 5. Target crown

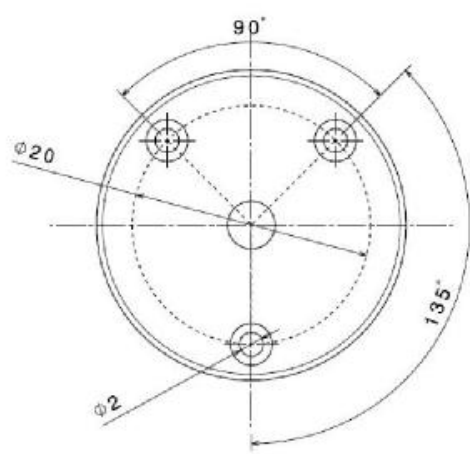

Fig. 6. Target dimensions

\subsection{Measuring Process}

As there are 135 points in the grid, automation is required, otherwise making the repeatability measurements take significant amount of manual work. Semi-automatic measuring process was built, but the limitations of the KMESS language do not allow fully automatic measuring process. CMM coordination system is first aligned manually teaching the corner positions of the grid. Process for the measurement of one point after alignment is following:

1. Robot is positioned to a point at matrix with its user interface

2. Automatic movement of the CMM to the measuring position

3. Manually clicking the approximate centre of the pre-alignment figure from the camera image at CMM interface

4. Automatic focus finding for Z-coordinate

5. Circle finding of the pre-alignment circle for the measurement origin (approximate centre of the robot axis)

6. Manually clicking the intersection of alignment line and circle to align the target coordinate system from the camera image

7. Automatic CMM positioning and finding all three balls using circle fitting tool.

8. Circle fitting to measured three ball centre positions

9. Saving the ball and fitted circle positions and radius in machine coordinate system. 
The measuring device is not touching the robot at any point of the process, however the rapid motions of the CMM table would cause some positioning deviation at the robot, thus speed of the CMM is reduced during the ball finding procedure. Quality of the measurement is manually controlled by following the reported ball and fitted circle diameters. $2 \mu \mathrm{m}$ variations are accepted for the $20 \mathrm{~mm}$ diameter circle fitting, otherwise the latest measurement is repeated.

In the first measurement round, all the grid points were measured once. This data was used to analyse the accuracy of the robot. Repeatability is the most used figure for comparing assembly robots, therefore the measurements should be repeated at least ten times around the same point by approaching the point from different directions. As the measurement process was very slow, only every second point was taken into account and each of these points was repeated only three times.

Reliability of the measuring method is tested by repeating same measurement five times in three farthest points on the grid. Sub micrometre deviation was found. Figure 8 presents the pre-alignment shape on the top of the axis and figure 8 presents the ruby ball visible in the camera image, together with circle finding search area.

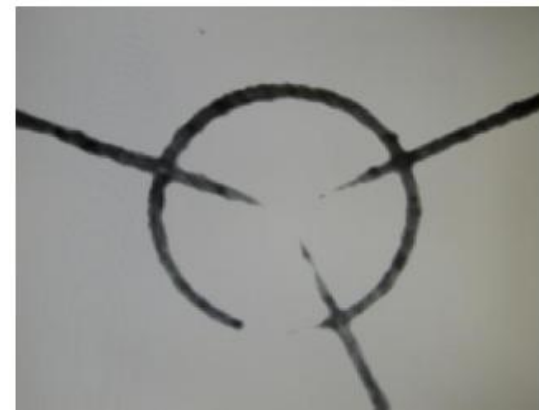

Fig. 7. Pre-alignment figure

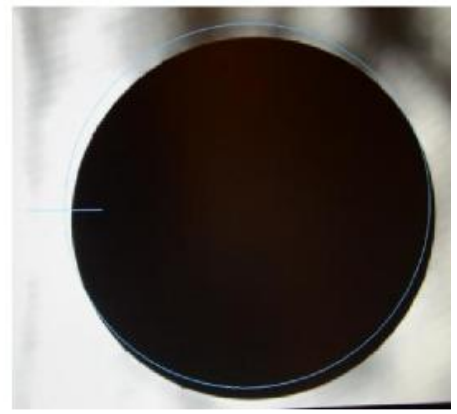

Fig. 8. 2 mm diameter ruby ball

\section{Measurement Results}

Measurement data is pre-processed using Matlab. As the data is in the machine coordinate system of the CMM, points are first transformed to coordinate system of the measurement setup. This is done by fitting the plane to cloud of points and executing corresponding offset and 3-axis rotations. Figure 9 presents the point cloud and the reference plane (ie. demanded positions) after the calibration. Several operations for the point data must be done to make it comparable with the corresponding reference points. As the step-size of the measurement grid is $7 \mathrm{~mm}$ it is straightforward to sort the point coordinates to corresponding $15 \mathrm{x} 9 \mathrm{X}-, \mathrm{Y}-$ and $\mathrm{Z}$ position matrices. 


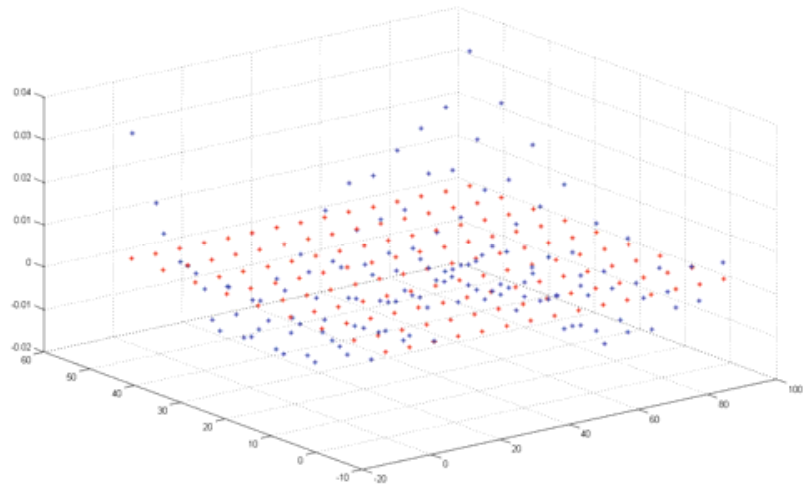

Fig. 9. Cloud of measured points (blue) and target points (red)

\subsection{Accuracy}

Accuracy of the robot is analysed according to the ISO-9283 standard [4]. Positional error is determined for each point by calculating the distance between the measured and the reference points. As the distance is always positive value, also comparison is made for each coordinate separately. Figure 10 presents the positioning errors for each dimension and the combined positioning error of the robot (note! XYcoordinates of the figures are point indexes. Zero point of the robot is at point $(7.5,-4)$ in the figure index coordinate system).
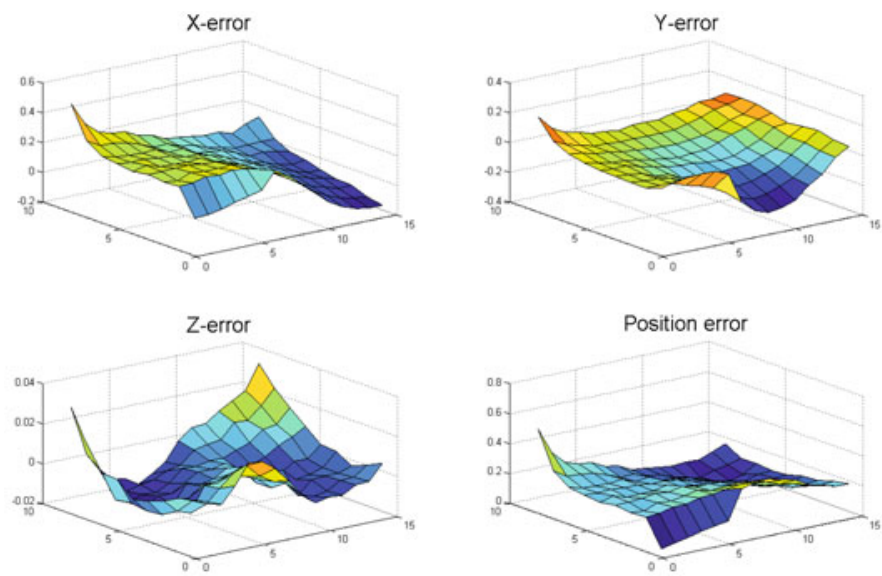

Fig. 10. Position errors in millimetres

It can be seen that there is a linear scaling error in X-direction and the corner positions have the worst accuracy. Also the $\mathrm{Y}$-direction has linear error as a function of distance from robot's zero position. Coloured error values forms a circular shapes on the surface map. Linear scaling could be calibrated by simply scaling the robot's coordinate system. Measured accuracy of the robot with and without unstable corner positions is presented on table 2 . 
Table 2. Positioning errors of the robot

Axis $\quad$ Error, without corners

Error, all positions

$\begin{array}{ccc}\text { X error } & -0,18 \ldots 0,2 \mathrm{~mm} & -0,18 \ldots 0,4 \mathrm{~mm} \\ \text { Y error } & -0,22 \ldots 0,08 \mathrm{~mm} & -0,22 \ldots 0,2 \mathrm{~mm} \\ \text { Z error } & -0,015 \ldots 0,02 \mathrm{~mm} & -0.015 \ldots 0.4 \mathrm{~mm} \\ \text { Positioning error } & 0.05 \ldots 0.3 \mathrm{~mm} & 0,05 \ldots 0,4 \mathrm{~mm}\end{array}$

\section{$5 \quad$ Conclusions and Future Work}

Optical measurement principle and method presented in this paper is proven to be suitable method for analysing the positioning accuracy and repeatability of the miniature robot systems. Method is suitable for desktop sized and smaller systems, which could be mounted inside the coordinate measuring machine. Repeatability of the measurement process itself must be verified. Deviation of the process should be ten times higher than expected accuracy. Total accuracy deviation for the measurement setup presented in this paper is around $10 \mu \mathrm{m}$ in $\mathrm{X}$ - and Y-directions. Zaccuracy deviation remains partially unknown as it is based on the automatic focus finding of the CMM, and deviation of it should be tested more carefully. Because of presented uncertainties this method is most suitable for analysing the positioning accuracy. If the expected accuracy of the robot is better than $5 \mu \mathrm{m}$ other methods should be considered.

Future work will contain a closer analysis of the measurement data and more comprehensive measurement series for the repeatability analysis. As the presented method has proven to be suitable method for calibrating robot's kinematics errors, more detailed calibration method for mini and micro size parallel robot will be studied. Measurement setup will be tested with four DOF version of the robot.

\section{References}

1. Vuola, A., Siltala, N., Heikkilä, R., Tuokko, R.: Micro H-scara robot: findings and results. In: Proceedings of the 7th International Workshop on Microfactories, IWMF 2010, Daejeon, Korea (2010)

2. Burisch, A., Wrege, J., Soetebier, S., Raatz, A., Hesselbach, J., Slatter, R.: "Parvus" a Micro-Parallel-Scara Robot for Desktop Assembly Lines. IFIP, vol. 198 (2006)

3. Hemming, B.: Measurement Traceability and Uncertainty in Machine Vision Applications. Mikes Metrology Publication J6/2007 (2007)

4. ISO 9283 Manipulating industrial robots - Performance criteria and related test methods 